\title{
Plasma free amino acid profiles evaluate risk of metabolic syndrome, diabetes, dyslipidemia, and hypertension in a large Asian population
}

Natsu Yamaguchi ${ }^{1 \dagger}$, MH Mahbub ${ }^{1 \dagger}$, Hidekazu Takahashi ${ }^{1}$, Ryosuke Hase ${ }^{1}$, Yasutaka Ishimaru ${ }^{1}$, Hiroshi Sunagawa ${ }^{1}$, Hiroki Amano ${ }^{2}$, Mikiko Kobayashi- Miura ${ }^{3}$, Hideyuki Kanda ${ }^{4}$, Yasuyuki Fujita ${ }^{4}$, Hiroshi Yamamoto ${ }^{5}$, Mai Yamamoto ${ }^{5}$, Shinya Kikuchi ${ }^{5}$, Atsuko Ikeda ${ }^{5}$, Mariko Takasu ${ }^{5}$, Naoko Kageyama ${ }^{5}$, Mina Nakamura ${ }^{5}$ and Tsuyoshi Tanabe ${ }^{1 *}$

\begin{abstract}
Background: Recently, the association of plasma free amino acid (PFAA) profile and lifestyle-related diseases has been reported. However, few studies have been reported in large Asian populations, about the usefulness of PFAAs for evaluating disease risks. We examined the ability of PFAA profiles to evaluate lifestyle-related diseases in so far the largest Asian population.

Methods: We examined plasma concentrations of 19 amino acids in 8589 Japanese subjects, and determined the association with variables associated with obesity, blood glucose, lipid, and blood pressure. We also evaluated the PFAA indexes that reflect visceral fat obesity and insulin resistance. The contribution of single PFAA level and relevant PFAA indexes was also examined in the risk assessment of lifestyle-related diseases.

Results: Of the 19 amino acids, branched-chain amino acids and aromatic amino acids showed association with obesity and lipid variables. The PFAA index related to visceral fat obesity showed relatively higher correlation with variables than that of any PFAA. In the evaluation of lifestyle-related disease risks, the odds ratios of the PFAA index related to visceral fat obesity or insulin resistance with the diseases were higher than most of those of individual amino acid levels even after adjusting for potential confounding factors. The association pattern of the indexes and PFAA with each lifestyle-related disease was distinct.
\end{abstract}

Conclusions: We confirmed the usefulness of PFAA profiles and indexes as markers for evaluating the risks of lifestyle-related diseases, including diabetes mellitus, metabolic syndrome, dyslipidemia, and hypertension in a large Asian population.

Keywords: Amino acids, Lifestyle-related diseases, Metabolic syndrome, Diabetes mellitus, Dyslipidemia, Hypertension

\section{Background}

Lifestyle-related diseases are pathophysiological states that include metabolic syndrome, diabetes mellitus $(\mathrm{DM})$, dyslipidemia, hypertension and gout, and often lead to cardiovascular disease $[1,2]$. Excess visceral fat and hyperinsulinemia are regarded as risk factors of

\footnotetext{
* Correspondence: tanabe@yamaguchi-u.ac.jp

${ }^{\dagger}$ Equal contributors

${ }^{1}$ Department of Public Health and Preventive Medicine, Graduate School of Medicine, Yamaguchi University, 1-1-1 Minami-Kogushi, Ube, Yamaguchi

755-8505, Japan

Full list of author information is available at the end of the article
}

lifestyle-related diseases [3, 4], and early detection of such factors is important for prevention of the diseases. Because of a shift towards the Western lifestyle and diet, the prevalence of lifestyle-related diseases has significantly increased in Asian populations [2]. For early detection of lifestyle-related diseases, the development of useful biomarkers is essential which would also help in better understanding of the disease pathophysiology and early intervention to avoid the progression of diseases and deterioration of patients' conditions [5].

Recent reports have shown that plasma free amino acid (PFAA) profile can serve as an effective biomarker 
for detection of lifestyle-related diseases [6-9]. PFAA profile was also reported to be useful for identifying diabetic patients at risk of developing cardiovascular diseases [10, 11]. Although these reports suggested that changes of amino acid metabolism play important roles in the pathogenesis of lifestyle-related diseases, the number of subjects in those studies was limited. Furthermore, we previously reported the construction of PFAA index 1 and index 2, which evaluated visceral fat area (VFA) and insulin resistance, respectively, and revealed their association with variables of lifestyle-related diseases and usefulness of such indexes for predicting the future risk of developing the diseases including metabolic syndrome, DM, dyslipidemia, and hypertension [12]. However, it is necessary to confirm the capabilities of the PFAA indexes to evaluate the risk of lifestylerelated diseases in a large Japanese population.

Therefore, the purpose of this study was to examine the ability of PFAA and relevant indexes for evaluating lifestyle-related diseases in so far the largest sample of an Asian population for such type of studies.

\section{Methods}

\section{Subjects}

The study was conducted in accordance with the Declaration of Helsinki, and the institutional review board of Shimane University (20100129-3) and Yamaguchi University (H25-26-2) approved the current study protocol. A total of 8589 Japanese subjects who underwent the health examination during 2009 to 2011 in Shimane prefecture and gave informed consent to participate in the study, were included.

\section{Measurement of metabolic variables and quantification of PFAAs}

Blood samples were taken from the subjects after an 8-h fast. Serum levels of total cholesterol (T-CHO), highdensity lipoprotein cholesterol (HDL-C), low- density lipoprotein cholesterol (LDL-C), and triglyceride (TG) were determined enzymatically. Free plasma glucose (FPG) was measured with the hexokinase method, and HbA1c was determined using the latex agglutination immunoassay. Plasma amino acid concentrations were analyzed following the protocol previously described elsewhere [12-16]. Briefly, blood samples (5 mL) were collected from forearm veins after overnight fasting into tubes containing disodium ethylenediaminetetraacetate that were immediately placed on ice. The plasma was prepared by centrifugation at 3000 r.p.m. at $4{ }^{\circ} \mathrm{C}$ for $15 \mathrm{~min}$ and then stored at $-80{ }^{\circ} \mathrm{C}$ until analysis. The plasma amino acid concentrations were measured by high-performance liquid chromatographyelectrospray ionization mass spectrometry followed by precolumn derivatization as previously described [13-16].
The following 19 amino acids were measured: Alanine (Ala), Arginine (Arg), Asparagine (Asn), Citrulline (Cit), Glutamine (Gln), Glycine (Gly), Histidine (His), Isoleucine (Ile), Leucine (Leu), Lysine (Lys), Methionine (Met), Ornithine (Orn), Phenylalanine (Phe), Proline (Pro), Serine (Ser), Threonine (Thr), Tryptophane (Trp), Tyrosine (Tyr), and Valine (Val). We did not perform the measurements of other genetically-encoded amino acids like glutamate, aspartate, and cysteine due to their instability in the blood [14].

\section{Clinical assessment}

In this study, metabolic syndrome was defined according to the following Japanese diagnostic criteria for the syndrome: visceral obesity (waist $\geq 85 \mathrm{~cm}$ in males and $\geq 90 \mathrm{~cm}$ in females) plus at least 2 of the following three components: (1) HDL-C $<40 \mathrm{mg} / \mathrm{dL}, \mathrm{TG} \geq 150 \mathrm{mg} / \mathrm{dL}$, or the use of medication for dyslipidemia; (2) FPG $\geq$ $110 \mathrm{mg} / \mathrm{dL}$ or the use of medication for DM; and (3) blood pressure $\geq 130 / 85 \mathrm{mmHg}$ or the use of antihypertensive medication. DM was defined in patients with FPG $\geq 126 \mathrm{mg} / \mathrm{dL}, \mathrm{HbA} 1 \mathrm{c} \geq 6.5 \%$, or those who were taking medication for DM. Dyslipidemia was defined in individuals with fasting LDL-C $\geq 140 \mathrm{mg} / \mathrm{dL}, \mathrm{HDL}-\mathrm{C}<$ $40 \mathrm{mg} / \mathrm{dL}, \mathrm{TG} \geq 150 \mathrm{mg} / \mathrm{dL}$, or those who were taking medication for dyslipidemia. Hypertension was defined in patients with systolic blood pressure (SBP) $\geq 140$ or diastolic blood pressure (DBP) $\geq 90 \mathrm{mmHg}$ or those who were taking antihypertensive medications.

\section{Calculation of PFAA indexes}

In this study, we used the amino acid index 1 and index 2 constructed and validated in a previous study [12]. The amino acid index 1 is the multiple linear regression model with variable selection to model the relationships between the PFAA profiles with the visceral fat area, consisting of Leu, Ala, Tyr, Asn, Trp, and Gly. The amino acid index 2 is the multiple linear regression model with variable selection to model the relationships between the PFAA profiles with 2-h post-challenge insulin levels (Ins120 min), consisting of Ile, Ala, Tyr, Phe, Met, and His. Therefore, each of these PFAA indexes (index 1 and index 2) is a single dimension that contains information on multidimensional PFAA profiles. Such compression of information on PFAA profiles allows maximization of the discrimination between patients and control subjects.

\section{Correlation between PFAAs and metabolic variables}

Correlation analysis between each of single PFAA concentration and PFAA indexes and metabolic variables was performed as previously described [12] by using the Pearson product-moment correlation coefficient. In addition, two-dimensional hierarchical cluster analysis 
that was based on the correlation coefficient matrix between the PFAA concentrations and additional measured variables was performed. For each single PFAA and the PFAA indexes, three different models were used with or without adjustments for variables as follows: model 1) without adjusting, model 2) adjusted for age and gender, model 3) adjusted for age, gender, and body mass index (BMI).

\section{Association between PFAAs and lifestyle related diseases} We examined the relationships of the PFAA profiles to lifestyle-related diseases to determine whether each PFAA index and single PFAA concentration were related to DM, metabolic syndrome, dyslipidemia, and hypertension. The PFAA indexes and all of the amino acids were scaled to multiples of 1 SD. A logistic regression analysis was used to assess the contribution of each PFAA index and single PFAA concentration as continuous variables in the evaluation of these diseases. The logistic regression analysis was performed with adjustment for age and gender. Furthermore, to exclude the cross-over effects among diseases on the single PFAA level and each PFAA index, further adjustments were performed as follows: metabolic syndrome for age, gender and BMI; DM for age, gender, BMI, LDL-C, HDL-C, TG, SBP and DBP; dyslipidemia for age, gender, BMI, FPG, HbA1c, SBP and DBP; hypertension for age, gender, BMI, FPG, HbA1c, LDL-C, HDL-C and TG.

A two-sided probability value of $p<0.01$ was considered to be statistically significant. $\mathrm{R}$ version 3.1.3 [ $\mathrm{R}$ Core Team (2015). R: A language and environment for statistical computing. R Foundation for Statistical Computing, Vienna, Austria] was used for the statistical analyses. All of the data were analyzed anonymously throughout the study.

\section{Results}

The numbers of subjects and their demographic and clinical characteristics have been presented in Table 1. Significant differences $(p<0.001$ to 0.01$)$ between the diseased and non-diseased populations were observed for all variables except for LDL cholesterol for DM and hypertension.

Amino acid concentrations in the study populations are shown in Table 2. Concentrations of PFAA differed significantly between the diseased and non-diseased subjects for metabolic syndrome, DM, dyslipidemia, and hypertension $(p<0.001$ to 0.05$)$. Overall, the concentrations of PFAA were higher in the diseased compared to the non-diseased; however, the concentrations of Gly and Ser for all diseases (except Ser for DM) and Asn for dyslipidemia showed the opposite trend.

We first examined the relationship between variables that were associated with lifestyle-related diseases and amino acids measured in this study (Table 3). The variables are obesity-related (BMI, waist circumference), glucose-related (FPG, HbA1c), lipid-related (HDL-C, LDL-C, and TG), and blood pressure-related (SBP, DBP). Branched-chain amino acids (BCAA; Val, Leu, Ile) showed moderately positive correlation with both obesity variables and with TG. All other correlations of BCAA with the rest variables were positive but weak. Like BCAA, aromatic amino acids (AAA; Tyr, Phe, Trp) also showed similar trends of correlations: positive (moderate to weak) correlation with both obesity variables and with TG, and weakly positive correlations with others. Among gluconeogenic amino acids (Ala, Gly, Gln, Ser), Ala showed moderately positive correlation with several variables including BMI, waist circumstance, blood glucose, and TG. Conversely, the correlation of Gly, Gln, and Ser was very weak or negative with almost all variables. Correlations of other single amino acids with lifestyle-related diseases were either very low or did not show any specific trend. Both PFAA indexes exhibited moderate to weak positive correlations with most of the variables associated with lifestyle-related diseases. Overall, the correlations were higher for the PFAA indexes compared with those for the single amino acids.

We then examined the capability of PFAA to evaluate the risk of metabolic syndrome, DM, dyslipidemia, and hypertension after adjustments for age and gender (Table 4). All the BCAA showed significant positive associations with all four diseases examined [OR between 1.23 and 2.16; 95\% CI between 1.15 and 1.98 (lower) and 1.30 to 2.36 (upper); $p<0.001$ ]. Especially, the odds ratios of BCAA with metabolic syndrome, DM and dyslipidemia were high, at least 1.7. Also, the odds ratios of AAA showed significant positive association with all four diseases [OR between 1.11 and 1.65; 95\% CI between 1.05 and 1.52 (lower) and 1.17 to 1.78 (upper); $p<0.001$ ] except Trp in DM. The odds ratios shown by AAA were relatively low compared with those by BCAA. Gluconeogenesis-related amino acids showed a typical pattern of odds ratio for all the four diseases, positive association for Ala [OR between 1.37 and 2.15; 95\% CI between 1.30 and 1.52 (lower) and 1.98 to 2.33 (upper); $p<0.001]$ and inverse association for Gly, Gln, and Ser [OR between 0.57 and 0.85 ; 95\% CI between 0.51 and 0.80 (lower) and 0.64 to 0.89 (upper); $p<0.001$ ] except for Ser in DM and Gln in dyslipidemia (Table 3). Then we confirmed the capabilities of the PFAA index 1 [OR between 1.52 and 3.23 ; 95\% CI between 1.44 and 2.92 (lower) and 1.61 to 3.57 (upper); $p<0.001$ ] and index 2 [OR between 1.48 and 2.37; 95\% CI between 1.39 and 2.16 (lower) and 1.57 to 2.61 (upper); $p<0.001$ ] to evaluate the significant positive association with lifestylerelated diseases (Table 4). The index 1 showed higher 
Table 1 Demographic and clinical characteristics of the study subjects. Values are expressed as means \pm standard deviations for continuous variables

\begin{tabular}{|c|c|c|c|c|c|c|c|c|}
\hline \multirow[b]{2}{*}{ Characteristics } & \multicolumn{2}{|c|}{ Metabolic syndrome } & \multicolumn{2}{|l|}{ DM } & \multicolumn{2}{|l|}{ Dyslipidemia } & \multicolumn{2}{|l|}{ Hypertension } \\
\hline & Disease & Non-disease & Disease & Non-disease & Disease & Non-disease & Disease & Non-disease \\
\hline $\mathrm{N}$, subjects & 737 & 6433 & 465 & 7650 & 3582 & 4533 & 3141 & 4974 \\
\hline (male, female) & $(539,198)$ & $(2915,3518)$ & $(284,181)$ & $(3555,4095)$ & $(1708,1874)$ & $(2131,2402)$ & $(1613,1528)$ & $(2226,2748)$ \\
\hline Age & $58.6 \pm 11.7$ & $52.7 \pm 15.3^{* * *}$ & $65.4 \pm 11.9$ & $55.9 \pm 16.8^{* * *}$ & $60.2 \pm 14.4$ & $53.5 \pm 17.7^{* * * *}$ & $65.1 \pm 13.0$ & $51.0 \pm 16.4^{* * *}$ \\
\hline BMI $\left(\mathrm{kg} / \mathrm{m}^{2}\right)$ & $26.9 \pm 3.1$ & $22.3 \pm 3.1^{* * *}$ & $24.2 \pm 3.8$ & $22.7 \pm 3.3^{* * *}$ & $23.6 \pm 3.3$ & $22.1 \pm 3.3^{* * *}$ & $23.7 \pm 3.5$ & $22.1 \pm 3.2^{* * *}$ \\
\hline Waist (cm) & $93.5 \pm 6.6$ & $79.7 \pm 8.6^{* * *}$ & $86.7 \pm 9.5$ & $80.8 \pm 9.3^{* * *}$ & $84.3 \pm 8.7$ & $78.7 \pm 9.2^{* * *}$ & $85.0 \pm 9.1$ & $79.1 \pm 8.9^{* * *}$ \\
\hline FPG (mg/dL) & $114 \pm 34$ & $95 \pm 13^{* * *}$ & $136 \pm 45$ & $94 \pm 10^{* * *}$ & $99 \pm 20$ & $95 \pm 15^{* * *}$ & $101 \pm 21$ & $94 \pm 15^{* * *}$ \\
\hline HbA1c (\%) & $6.3 \pm 1.2$ & $5.6 \pm 0.5^{* * *}$ & $7.3 \pm 1.3$ & $5.6 \pm 0.4^{* * *}$ & $5.9 \pm 0.8$ & $5.6 \pm 0.5^{* * *}$ & $5.9 \pm 0.8$ & $5.6 \pm 0.6^{* * *}$ \\
\hline TG (mg/dL) & $170 \pm 102$ & $93 \pm 61^{* * *}$ & $128 \pm 101$ & $98 \pm 65^{* * *}$ & $133 \pm 86$ & $74 \pm 28^{* * *}$ & $113 \pm 76$ & $92 \pm 60^{* * *}$ \\
\hline $\mathrm{HDL}-\mathrm{C}(\mathrm{mg} / \mathrm{dL})$ & $54 \pm 15$ & $66 \pm 17^{* * *}$ & $58 \pm 16$ & $65 \pm 17^{* * *}$ & $59 \pm 16$ & $69 \pm 16^{* * *}$ & $62 \pm 16$ & $66 \pm 17^{* * *}$ \\
\hline $\mathrm{LDL}-\mathrm{C}(\mathrm{mg} / \mathrm{dL})$ & $125 \pm 32$ & $121 \pm 31^{* * *}$ & $119 \pm 32$ & $120 \pm 30$ & $137 \pm 33$ & $107 \pm 20^{* * *}$ & $121 \pm 30$ & $120 \pm 31$ \\
\hline $\mathrm{SBP}(\mathrm{mmHg})$ & $140 \pm 15$ & $125 \pm 16^{* * *}$ & $135 \pm 18$ & $127 \pm 17^{* * *}$ & $131 \pm 17$ & $124 \pm 16^{* * *}$ & $141 \pm 16$ & $119 \pm 11^{* * *}$ \\
\hline $\mathrm{DBP}(\mathrm{mmHg})$ & $87 \pm 10$ & $77 \pm 11^{* * *}$ & $79 \pm 12$ & $78 \pm 11^{* *}$ & $79 \pm 11$ & $76 \pm 11^{* * *}$ & $84 \pm 12$ & $73 \pm 9^{* * *}$ \\
\hline
\end{tabular}

$B M I$ body mass index, FPG Free plasma glucose, $H b A 1 c$ haemoglobin $A 1 c, T G$ triglyceride, $H D L-C$ high-density lipoprotein cholesterol, $L D L-C$ low-density lipoprotein cholesterol, SBP systolic blood pressure, DBP diastolic blood pressure

Differences in the total number of total subjects for different diseases are due to varying missing data

Statistical differences between disease and non-disease subjects are shown as ${ }^{* *} p<0.01$, and ${ }^{* * *} p<0.001$, respectively

Table 2 Plasma free amino acid concentrations ( $\mu \mathrm{mol} / \mathrm{L}$ ) and index values in the study population. The values are shown as means \pm standard deviations

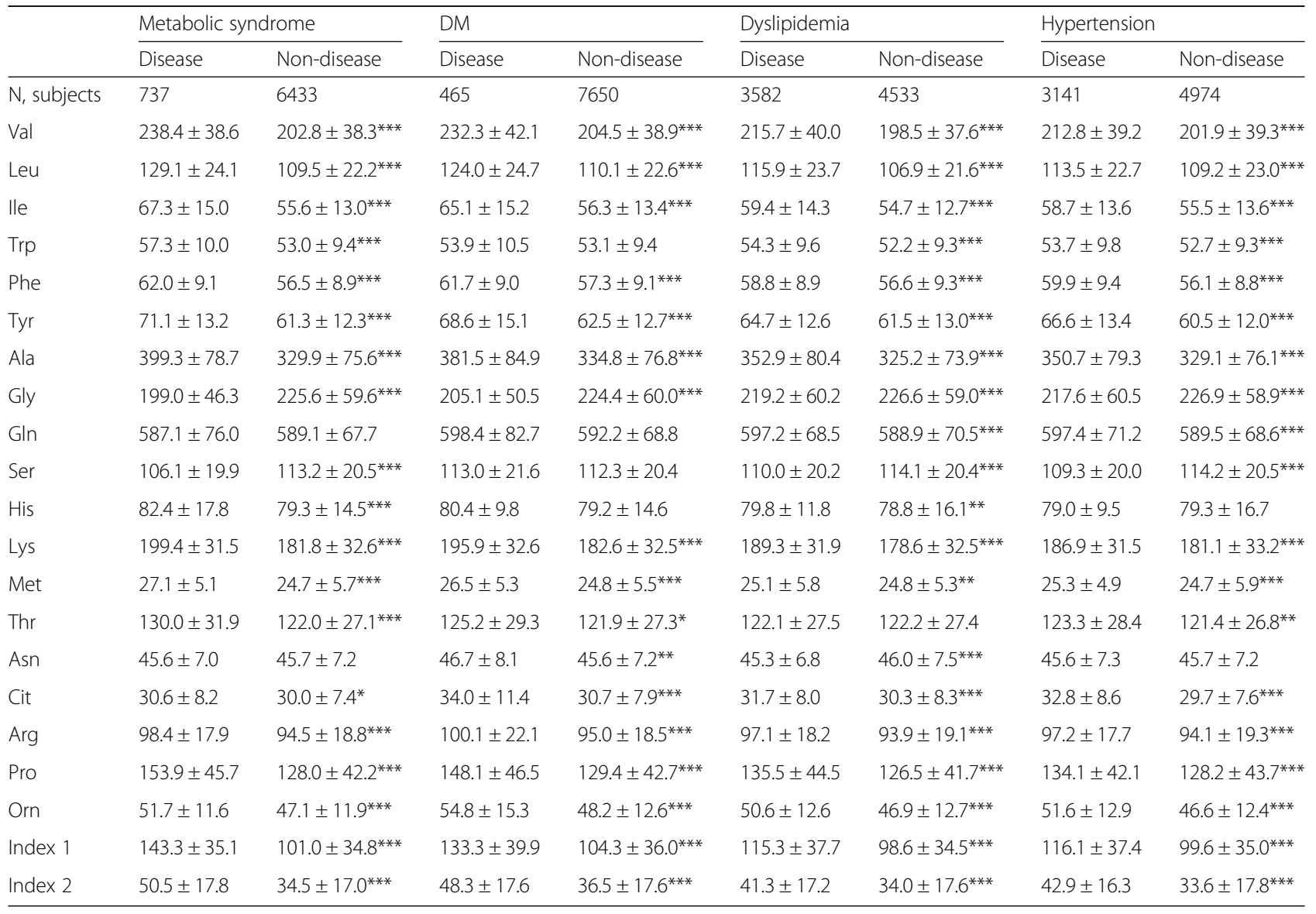


Table 3 Correlation between PFAA profiles and metabolic variables

\begin{tabular}{|c|c|c|c|c|c|c|c|c|c|}
\hline & & Obesity & & Glucose & & Lipid & & Blood pr & \\
\hline & Age & $\mathrm{BMI}$ & Waist circumstance & FPG & $\mathrm{HbA1c}$ & $\mathrm{LDL}$ & TG & SBP & DBP \\
\hline Val & 0.008 & 0.391 & 0.406 & 0.247 & 0.199 & 0.142 & 0.341 & 0.151 & 0.190 \\
\hline Leu & -0.052 & 0.371 & 0.377 & 0.223 & 0.162 & 0.117 & 0.340 & 0.109 & 0.198 \\
\hline lle & -0.035 & 0.327 & 0.336 & 0.219 & 0.162 & 0.056 & 0.353 & 0.123 & 0.184 \\
\hline Trp & -0.064 & 0.219 & 0.230 & 0.109 & 0.033 & 0.045 & 0.254 & 0.076 & 0.173 \\
\hline Phe & 0.278 & 0.255 & 0.309 & 0.177 & 0.200 & 0.028 & 0.156 & 0.176 & 0.140 \\
\hline Tyr & 0.282 & 0.325 & 0.393 & 0.212 & 0.183 & 0.028 & 0.201 & 0.233 & 0.195 \\
\hline Ala & -0.030 & 0.331 & 0.337 & 0.300 & 0.171 & 0.089 & 0.328 & 0.183 & 0.226 \\
\hline Gly & 0.035 & -0.174 & -0.183 & -0.108 & -0.088 & -0.039 & -0.163 & -0.084 & -0.125 \\
\hline Gln & 0.243 & 0.033 & 0.075 & 0.001 & 0.072 & 0.054 & -0.075 & 0.044 & -0.019 \\
\hline Ser & -0.051 & -0.136 & -0.175 & -0.047 & -0.040 & -0.061 & -0.264 & -0.144 & -0.156 \\
\hline $\mathrm{His}$ & -0.062 & 0.121 & 0.118 & 0.063 & 0.006 & 0.027 & 0.118 & 0.010 & 0.079 \\
\hline Lys & 0.108 & 0.219 & 0.262 & 0.181 & 0.157 & 0.123 & 0.158 & 0.106 & 0.140 \\
\hline Met & -0.012 & 0.166 & 0.172 & 0.151 & 0.046 & -0.059 & 0.116 & 0.069 & 0.133 \\
\hline Thr & -0.080 & 0.102 & 0.088 & 0.087 & -0.015 & -0.125 & 0.071 & 0.052 & 0.114 \\
\hline Asn & 0.008 & -0.014 & -0.010 & 0.064 & 0.000 & -0.091 & -0.017 & 0.000 & 0.035 \\
\hline Cit & 0.460 & -0.104 & -0.017 & 0.060 & 0.176 & -0.034 & 0.018 & 0.139 & -0.009 \\
\hline Arg & 0.141 & 0.047 & 0.094 & 0.146 & 0.122 & 0.063 & 0.047 & 0.091 & 0.093 \\
\hline Pro & -0.073 & 0.200 & 0.204 & 0.160 & 0.080 & -0.002 & 0.235 & 0.078 & 0.129 \\
\hline Orn & 0.376 & 0.091 & 0.171 & 0.151 & 0.200 & 0.019 & 0.104 & 0.166 & 0.057 \\
\hline Index 1 & 0.093 & 0.463 & 0.494 & 0.310 & 0.252 & 0.135 & 0.379 & 0.242 & 0.261 \\
\hline Index 2 & 0.255 & 0.352 & 0.403 & 0.249 & 0.259 & 0.097 & 0.291 & 0.247 & 0.187 \\
\hline N, subjects & 8588 & 8589 & 7170 & 7324 & 8004 & 8023 & 8023 & 8115 & 8115 \\
\hline
\end{tabular}

Pearson's correlation coefficients were calculated, and hierarchical clustering was conducted $B M I$ Body mass index, FPG Fasting plasma glucose, HbA1c Hemoglobin A1c, LDL-CHO LDL cholesterol, TG Triglyceride, SBP Systolic blood pressure, DBP Diastolic blood pressure

index 1 and index 2: the Indexes generated by plasma free amino acid profiles

odds ratio than that of any amino acid with metabolic syndrome, DM, and hypertension. The index 2 showed higher odds ratio than that of each amino acid with metabolic syndrome and hypertension.

Next we confirmed the capabilities of the PFAA to evaluate their association with lifestyle-related diseases after excluding the probable cross-over effects among diseases on the single PFAA level and each PFAA index (Table 5). For this purpose, the diseases were adjusted for different relevant factors for each disease separately. After such adjustments, the typical pattern of association for BCAA persisted with all diseases except hypertension. In contrast, after adjustments, the odds ratios of AAA lost significant positive associations for all diseases except Trp in dyslipidemia. On the other hand, most of the gluconeogenesis-related amino acids retained their typical pattern of association with the diseases. Also, positive associations of both PFAA indexes remained statistically significant with all lifestyle-related diseases (Table 5).

\section{Discussion}

In this study, we have examined the relationship between PFAA profiles and lifestyle-related disease risks with more than 8000 subjects, so far the largest Asian population for such type of studies. We confirmed that PFAA profiles and the indexes generated from them are useful for evaluating lifestyle-related diseases. Our analysis showed that concentrations of several single PFAA and PFAA indexes could identify the subjects who are at risks for metabolic syndrome, DM, dyslipidemia, and hypertension.

Recently, an increased number of reports on the association between PFAA profiles and lifestyle-related diseases are being observed [11-13]. For example, BCAAs especially Val and Leu, have been proposed as a cardiometabolic risk marker independent of BMI category [11]. Furthermore, BCAAs have been linked to the metabolic syndrome, insulin resistance or type $2 \mathrm{DM}[12,17]$. Interactions of excess BCAA and lipids may lead to the development of $\beta$-cell dysfunction, which drives the transition from the obese, insulin-resistant state to type 
Table 4 The odds ratios for evaluating metabolic syndrome, DM, dyslipidemia, or hypertension adjusting for age and gender

\begin{tabular}{|c|c|c|c|c|c|c|c|c|c|c|c|c|}
\hline \multirow[b]{3}{*}{ Val } & \multicolumn{3}{|c|}{ Metabolic syndrome } & \multicolumn{3}{|l|}{$\mathrm{DM}$} & \multicolumn{3}{|c|}{ Dyslipidemia } & \multicolumn{3}{|c|}{ Hypertension } \\
\hline & \multicolumn{2}{|c|}{ Odds ratio } & \multirow{2}{*}{$\begin{array}{c}p \text {-value } \\
<0.001\end{array}$} & \multicolumn{2}{|c|}{ Odds ratio } & \multirow{2}{*}{$\begin{array}{l}p \text {-value } \\
<0.001\end{array}$} & \multicolumn{2}{|c|}{ Odds ratio } & \multirow{2}{*}{$\begin{array}{l}p \text {-value } \\
<0.001\end{array}$} & \multicolumn{2}{|c|}{ Odds ratio } & \multirow{2}{*}{$\begin{array}{l}p \text {-value } \\
<0.001\end{array}$} \\
\hline & 2.16 & $(1.98-2.36)$ & & 1.91 & $(1.73-2.11)$ & & 1.79 & $(1.69-1.89)$ & & 1.30 & $(1.23-1.37)$ & \\
\hline Leu & 2.04 & $(1.86-2.23)$ & $<0.001$ & 1.82 & $(1.63-2.02)$ & $<0.001$ & 1.88 & $(1.77-2.00)$ & $<0.001$ & 1.23 & $(1.15-1.30)$ & $<0.001$ \\
\hline Ile & 1.97 & $(1.80-2.15)$ & $<0.001$ & 1.75 & $(1.58-1.93)$ & $<0.001$ & 1.69 & $(1.59-1.79)$ & $<0.001$ & 1.27 & $(1.20-1.35)$ & $<0.001$ \\
\hline Trp & 1.27 & $(1.17-1.37)$ & $<0.001$ & 0.99 & $(0.89-1.10)$ & 0.859 & 1.36 & $(1.29-1.43)$ & $<0.001$ & 1.11 & $(1.05-1.17)$ & $<0.001$ \\
\hline Phe & 1.34 & $(1.24-1.45)$ & $<0.001$ & 1.20 & $(1.10-1.31)$ & $<0.001$ & 1.15 & $(1.10-1.21)$ & $<0.001$ & 1.13 & $(1.07-1.20)$ & $<0.001$ \\
\hline Tyr & 1.65 & $(1.52-1.78)$ & $<0.001$ & 1.24 & $(1.13-1.36)$ & $<0.001$ & 1.16 & $(1.10-1.22)$ & $<0.001$ & 1.25 & $(1.19-1.32)$ & $<0.001$ \\
\hline Ala & 2.15 & $(1.98-2.33)$ & $<0.001$ & 1.70 & $(1.55-1.86)$ & $<0.001$ & 1.53 & $(1.45-1.61)$ & $<0.001$ & 1.37 & $(1.30-1.45)$ & $<0.001$ \\
\hline Gly & 0.57 & $(0.51-0.64)$ & $<0.001$ & 0.69 & $(0.61-0.78)$ & $<0.001$ & 0.87 & $(0.83-0.91)$ & $<0.001$ & 0.84 & $(0.80-0.89)$ & $<0.001$ \\
\hline Gln & 0.83 & $(0.77-0.90)$ & $<0.001$ & 0.92 & $(0.83-1.01)$ & 0.082 & 1.01 & $(0.97-1.06)$ & 0.581 & 0.85 & $(0.80-0.89)$ & $<0.001$ \\
\hline Ser & 0.76 & $(0.70-0.83)$ & $<0.001$ & 1.14 & $(1.04-1.26)$ & 0.008 & 0.83 & $(0.79-0.87)$ & $<0.001$ & 0.80 & $(0.76-0.85)$ & $<0.001$ \\
\hline His & 1.06 & $(1.01-1.13)$ & 0.031 & 1.05 & $(0.99-1.11)$ & 0.134 & 1.17 & $(1.09-1.25)$ & $<0.001$ & 0.99 & $(0.94-1.05)$ & 0.739 \\
\hline Lys & 1.35 & $(1.25-1.47)$ & $<0.001$ & 1.30 & $(1.19-1.43)$ & $<0.001$ & 1.39 & $(1.32-1.47)$ & $<0.001$ & 1.02 & $(0.97-1.08)$ & 0.398 \\
\hline Met & 1.16 & $(1.07-1.26)$ & $<0.001$ & 1.13 & $(1.05-1.21)$ & 0.002 & 1.06 & $(1.00-1.11)$ & 0.038 & 1.03 & $(0.97-1.08)$ & 0.326 \\
\hline Thr & 1.18 & $(1.10-1.28)$ & $<0.001$ & 1.10 & $(1.00-1.21)$ & 0.042 & 1.02 & $(0.97-1.07)$ & 0.404 & 1.12 & $(1.07-1.18)$ & $<0.001$ \\
\hline Asn & 0.79 & $(0.72-0.86)$ & $<0.001$ & 1.05 & $(0.95-1.15)$ & 0.321 & 0.87 & $(0.83-0.91)$ & $<0.001$ & 0.89 & $(0.84-0.93)$ & $<0.001$ \\
\hline Cit & 0.84 & $(0.77-0.93)$ & $<0.001$ & 1.10 & $(1.00-1.20)$ & 0.053 & 0.97 & $(0.92-1.01)$ & 0.160 & 0.93 & $(0.88-0.99)$ & 0.013 \\
\hline Arg & 1.00 & $(0.92-1.08)$ & 0.993 & 1.13 & $(1.03-1.24)$ & 0.009 & 1.12 & $(1.07-1.17)$ & $<0.001$ & 0.98 & $(0.93-1.03)$ & 0.482 \\
\hline Pro & 1.46 & $(1.36-1.57)$ & $<0.001$ & 1.34 & $(1.24-1.46)$ & $<0.001$ & 1.31 & $(1.25-1.38)$ & $<0.001$ & 1.17 & $(1.11-1.24)$ & $<0.001$ \\
\hline Orn & 1.16 & $(1.07-1.26)$ & $<0.001$ & 1.23 & $(1.14-1.33)$ & $<0.001$ & 1.16 & $(1.11-1.22)$ & $<0.001$ & 1.04 & $(0.98-1.09)$ & 0.205 \\
\hline Index 1 & 3.23 & $(2.92-3.57)$ & $<0.001$ & 2.10 & (1.89-2.33) & $<0.001$ & 1.69 & $(1.61-1.79)$ & $<0.001$ & 1.52 & $(1.44-1.61)$ & $<0.001$ \\
\hline Index 2 & 2.37 & $(2.16-2.61)$ & $<0.001$ & 1.66 & $(1.50-1.84)$ & $<0.001$ & 1.53 & $(1.45-1.62)$ & $<0.001$ & 1.48 & $(1.39-1.57)$ & $<0.001$ \\
\hline
\end{tabular}

2 DM [17]. With respect to such factors like insulin sensitivity and visceral obesity, there are differences between Asian and Western populations $[18,19]$. But the number of studies focusing on these issues is limited [12, 20].

As revealed in this study, there was a consistent increase in the concentrations of BCAA in all four lifestyle-related diseases. Also, BCAA exhibited significant positive association with all those diseases. Such an observation is consistent with the findings of other research works $[12,13]$. As the underlying mechanisms, it has been suggested that a decrease in insulin activity and utilization of amino acids in muscles induces reduced uptake of BCAAs into muscles resulting in increased BCAA levels in lifestyle-related diseases [21]. Newgard et al. postulated that the rise in circulating BCAA in obese and insulin-resistant subjects is partially caused by a decline in their catabolism in adipose tissue possibly via down-regulation of the BCAA catabolic enzymes through the suppression of peroxisome proliferator-activated receptor- $\gamma$ (PPAR- $\gamma$ ) signaling in such metabolic adaptation [17]. All these might have been reflected in our findings of significant positive association of BCAA with lifestyle-related diseases. However, although metabolic syndrome, DM, dyslipidemia, and hypertension showed positive association with BCAA in this study after adjusting for age and gender, hypertension lost the association after further adjustments for the disease-specific factors. These results might suggest that in addition to the currently known risk factors like diet or nutrition, differing metabolism for different amino acids probably contributes to increased risk of lifestylerelated diseases.

The underlying mechanisms for the observed elevation in AAA concentrations remain unclear. However, repression of tyrosine aminotransferase during states of insulin resistance and DM may result in the increased levels of circulating Tyr and Phe as observed in our study [12, 22].

In our analysis, metabolic syndrome, dyslipidemia, and hypertension showed similar patterns for association with gluconeogenesis-related amino acids. The diseases positively correlated with Ala, and negatively with Gly, Gln and Ser except Ser in DM and Gln in dyslipidemia. It was reported that under condition of hyperinsulinemia, increased protein turnover causes greater supply of gluconeogenic amino acids to the liver, leading to the reductions of Gly, Gln and Ser [23]. Additionally, gluconeogenesis precursors including Ala have been reported to rise in those with deteriorating glucose tolerance [24]. 
Table 5 The odds ratios for evaluating metabolic syndrome, DM, dyslipidemia, or hypertension adjusting for additional factors

\begin{tabular}{|c|c|c|c|c|c|c|c|c|c|c|c|c|}
\hline \multirow[b]{3}{*}{ Val } & \multicolumn{3}{|c|}{${ }^{\mathrm{a} M e t a b o l i c ~ s y n d r o m e}$} & \multicolumn{3}{|c|}{${ }^{\mathrm{b} D M}$} & \multicolumn{3}{|c|}{${ }^{\mathrm{c} D y s l i p i d e m i a}$} & \multicolumn{3}{|c|}{ dHypertension } \\
\hline & \multicolumn{2}{|c|}{ Odds ratio } & \multirow{2}{*}{$\frac{p \text {-value }}{<0.001}$} & \multicolumn{2}{|c|}{ Odds ratio } & \multirow{2}{*}{$\begin{array}{l}p \text {-value } \\
<0.001\end{array}$} & \multicolumn{2}{|c|}{ Odds ratio } & \multirow{2}{*}{$\begin{array}{l}p \text {-value } \\
<0.001\end{array}$} & \multicolumn{2}{|c|}{ Odds ratio } & \multirow{2}{*}{$\frac{p \text {-value }}{0.816}$} \\
\hline & 1.48 & $(1.33-1.64)$ & & 1.67 & $(1.50-1.87)$ & & 1.48 & $(1.39-1.58)$ & & 1.01 & $(0.94-1.08)$ & \\
\hline Leu & 1.39 & $(1.25-1.54)$ & $<0.001$ & 1.59 & $(1.41-1.78)$ & $<0.001$ & 1.57 & $(1.47-1.68)$ & $<0.001$ & 0.95 & $(0.88-1.02)$ & 0.137 \\
\hline Ile & 1.46 & $(1.32-1.62)$ & $<0.001$ & 1.52 & $(1.36-1.69)$ & $<0.001$ & 1.43 & $(1.34-1.53)$ & $<0.001$ & 1.03 & $(0.96-1.11)$ & 0.414 \\
\hline Trp & 1.07 & $(0.97-1.18)$ & 0.169 & 0.88 & $(0.79-0.98)$ & 0.024 & 1.25 & $(1.18-1.32)$ & $<0.001$ & 0.98 & $(0.92-1.04)$ & 0.471 \\
\hline Phe & 0.96 & $(0.87-1.07)$ & 0.470 & 1.10 & $(1.00-1.21)$ & 0.051 & 1.01 & $(0.95-1.07)$ & 0.794 & 0.98 & $(0.92-1.04)$ & 0.503 \\
\hline Tyr & 1.08 & $(0.98-1.19)$ & 0.115 & 1.07 & $(0.97-1.18)$ & 0.164 & 0.96 & $(0.91-1.02)$ & 0.151 & 1.01 & $(0.95-1.08)$ & 0.740 \\
\hline Ala & 1.70 & $(1.55-1.87)$ & $<0.001$ & 1.47 & $(1.33-1.63)$ & $<0.001$ & 1.34 & $(1.26-1.42)$ & $<0.001$ & 1.08 & $(1.01-1.15)$ & 0.021 \\
\hline Gly & 0.76 & $(0.67-0.86)$ & $<0.001$ & 0.76 & $(0.67-0.86)$ & $<0.001$ & 0.96 & $(0.91-1.01)$ & 0.079 & 0.93 & $(0.88-0.99)$ & 0.020 \\
\hline Gln & 0.77 & $(0.70-0.84)$ & $<0.001$ & 0.93 & $(0.84-1.03)$ & 0.161 & 1.01 & $(0.96-1.06)$ & 0.688 & 0.89 & $(0.84-0.94)$ & $<0.001$ \\
\hline Ser & 0.82 & $(0.74-0.91)$ & $<0.001$ & 1.27 & $(1.14-1.40)$ & $<0.001$ & 0.88 & $(0.84-0.93)$ & $<0.001$ & 0.87 & $(0.82-0.93)$ & $<0.001$ \\
\hline His & 1.05 & $(0.98-1.13)$ & 0.137 & 1.03 & $(0.95-1.12)$ & 0.432 & 1.05 & $(0.99-1.11)$ & 0.109 & 0.89 & $(0.81-0.97)$ & 0.007 \\
\hline Lys & 1.14 & $(1.04-1.26)$ & 0.005 & 1.24 & $(1.12-1.36)$ & $<0.001$ & 1.25 & $(1.18-1.32)$ & $<0.001$ & 0.91 & $(0.86-0.97)$ & 0.002 \\
\hline Met & 1.04 & $(0.97-1.11)$ & 0.328 & 1.09 & $(1.02-1.17)$ & 0.017 & 0.98 & $(0.93-1.04)$ & 0.593 & 0.95 & $(0.89-1.02)$ & 0.127 \\
\hline Thr & 1.17 & $(1.08-1.27)$ & $<0.001$ & 1.05 & $(0.95-1.15)$ & 0.350 & 0.98 & $(0.93-1.03)$ & 0.421 & 1.06 & $(1.00-1.12)$ & 0.061 \\
\hline Asn & 0.84 & $(0.76-0.92)$ & $<0.001$ & 1.08 & $(0.98-1.19)$ & 0.103 & 0.90 & $(0.85-0.95)$ & $<0.001$ & 0.92 & $(0.87-0.98)$ & 0.008 \\
\hline Cit & 0.98 & $(0.88-1.08)$ & 0.648 & 1.13 & $(1.03-1.24)$ & 0.011 & 1.02 & $(0.97-1.08)$ & 0.463 & 1.04 & $(0.98-1.10)$ & 0.253 \\
\hline Arg & 1.01 & $(0.92-1.11)$ & 0.824 & 1.15 & $(1.05-1.27)$ & 0.003 & 1.11 & $(1.05-1.16)$ & $<0.001$ & 0.99 & $(0.93-1.05)$ & 0.658 \\
\hline Pro & 1.38 & $(1.27-1.50)$ & $<0.001$ & 1.25 & $(1.15-1.36)$ & $<0.001$ & 1.20 & $(1.14-1.27)$ & $<0.001$ & 1.05 & $(0.99-1.12)$ & 0.137 \\
\hline Orn & 1.06 & $(0.97-1.17)$ & 0.217 & 1.18 & $(1.09-1.29)$ & $<0.001$ & 1.11 & $(1.05-1.18)$ & $<0.001$ & 0.99 & $(0.93-1.05)$ & 0.716 \\
\hline Index 1 & 1.88 & $(1.68-2.11)$ & $<0.001$ & 1.83 & $(1.62-2.06)$ & $<0.001$ & 1.36 & $(1.28-1.45)$ & $<0.001$ & 1.12 & $(1.04-1.20)$ & 0.003 \\
\hline Index 2 & 1.44 & $(1.29-1.60)$ & $<0.001$ & 1.37 & $(1.22-1.53)$ & $<0.001$ & 1.24 & $(1.16-1.33)$ & $<0.001$ & 1.12 & $(1.04-1.21)$ & 0.002 \\
\hline
\end{tabular}

The present study has confirmed that PFAA indexes which reflect VFA and insulin resistance, respectively [12], could assess lifestyle-related diseases investigated in the largest Japanese population. In this study, index 1 was associated with most of the variables more positively than any single amino acid, and the tendency was more apparent than that of index 2 . The association pattern of the indexes and PFAA with each lifestyle-related disease is distinct. Although hypertension showed no association with BCAA and AAA after adjustments for exclusion of cross-over effects among diseases, corresponding odds ratios for both index 1 and index 2 were significant. Of the diseases examined, only dyslipidemia showed higher odds ratios with BCAA than with index 1 . These results revealed the distinct contribution of amino acid metabolism to the risk of each lifestyle-related disease. Further analysis of the relationship between the amino acid metabolisms and disease risk may lead to the understanding of pathophysiology, diagnosis and prevention of lifestylerelated diseases.
Our analysis showed moderate correlation between PFAA indexes and hypertension, which was previously shown to be weak [12]. The difference between the results might be due to a higher number of subjects in this study. However, it would be interesting to know the effects of salt intake on the relationship between hypertension and PFAA profiles in future studies.

The findings of our study should be interpreted in light of the limitation that we could not include the information related to the lifestyle factors such as physical activity and diet due to limited available data on these, which may have affected the PFAA profiles observed in this study.

\section{Conclusions}

Our study confirmed the usefulness of PFAA profiles as markers for evaluating the risks of lifestyle-related diseases, including DM, metabolic syndrome, dyslipidemia, and hypertension in a large Asian population. 


\section{Abbreviations}

AAA: Aromatic amino acids; Ala: Alanine; Arg: Arginine; Asn: Asparagine; BCAA: Branched-chain amino acids; BMl: Body mass index; Cit: Citrulline; DBP: Diastolic blood pressure; DM: Diabetes mellitus; FPG: Free plasma glucose; Gln: Glutamine; Gly: Glycine; HbA1c: Haemoglobin A1c; HDL-C: High-density lipoprotein cholesterol; His: Histidine; Ile: Isoleucine; LDL-C: Low-density lipoprotein cholesterol; Leu: Leucine; Lys: Lysine; Met: Methionine; Orn: Ornithine; PFAA: Plasma free amino acid; Phe: Phenylalanine; Pro: Proline: SBP: Systolic blood pressure; Ser: Serine; T-CHO: Cholesterol; TG: Triglyceride; Thr: Threonine; Trp: Tryptophane; Tyr: Tyrosine; Val: Valine; VFA: Visceral fat area

\section{Acknowledgements}

Not applicable

\section{Availability of data and materials}

Please contact the corresponding author for data requests.

\section{Authors' contributions}

NY, YF, HA, MKM, HY and TT designed the study. NY, MM, HT, RH, YI, HS, HA, MKM, HK, YF, HY and TT were involved in obtaining and surveying data. NY, MM, HT, YF, HY, MY, SK, Al, MT, NK, MN and TT analyzed the data and performed statistical analysis. All authors read and approved the final manuscript.

\section{Competing interests}

HY, MY, SK, Al, MT, NK, and MN are employees of Ajinomoto Co., Inc. TT, HA, and YF received research grants from Ajinomoto Co., Inc. This does not alter the authors' adherences to all of the journal policies. No other potential conflicts of interest in relation to this article are declared.

\section{Consent for publication}

Not applicable.

\section{Ethics approval and consent to participate}

The study was conducted in accordance with the Declaration of Helsinki, and the institutional review board of Shimane University (20100129-3) and Yamaguchi University (H25-26-2) approved the current study protocol. A total of 8589 Japanese subjects who underwent the health examination during 2009 to 2011 in Shimane prefecture and gave informed consent to participate in the study, were included.

\section{Author details}

${ }^{1}$ Department of Public Health and Preventive Medicine, Graduate School of Medicine, Yamaguchi University, 1-1-1 Minami-Kogushi, Ube, Yamaguchi 755-8505, Japan. ${ }^{2}$ Division of Health Administration and Promotion, Graduate School of Medicine, Tottori University, Yonago, Japan. ${ }^{3}$ Department of Biochemistry, Shimane University Faculty of Medicine, Izumo, Japan. ${ }^{4}$ Department of Environmental Medicine and Public Health, Faculty of Medicine, Shimane University, Izumo, Japan. ${ }^{5}$ Institute for Innovation, Ajinomoto Co., Inc., Kawasaki, Japan.

Received: 17 January 2017 Accepted: 1 February 2017

\section{Published online: 07 April 2017}

\section{References}

1. Tenenbaum A, Motro M, Schwammenthal E, Fisman E. Macrovascular complications of metabolic syndrome: an early intervention is imperative. Int J Cardiol. 2004;97:167-72.

2. Ma RC, Chan JC. Type 2 diabetes in East Asians: similarities and differences with populations in Europe and the United States. Ann N Y Acad Sci. 2013;1281:64-91.

3. Despres JP, Lemieux I. Abdominal obesity and metabolic syndrome. Nature. 2006:444:881-7

4. Van Gaal LF, Mertens IL, De Block CE. Mechanisms linking obesity with cardiovascular disease. Nature. 2006;444:875-80.

5. Imaizumi A, Nishikawa N, Yoshida H, Yoneda J, Takahena S, Takahashi M, et al. Clinical implementation of metabolomics. In: Roessner U, editor. Metabolomics. Rijeka: InTech; 2012. p. 289-314.

6. McCormack SE, Shaham O, McCarthy MA, Deik AA, Wang TJ, Gerszten RE, Clish CB, Mootha VK, Grinspoon SK, Fleischman A. Circulating branchedchain amino acid concentrations are associated with obesity and future insulin resistance in children and adolescents. Pediatr Obes. 2013;8:52-61.
7. Würtz $P$, Mäkinen VP, Soininen $P$, Kangas A, Tukiainen T, Kettunen J, et al. Metabolic signatures of insulin resistance in 7,098 young adults. Diabetes. 2012;61:1372-80.

8. Würtz $P$, Soininen $P$, Kangas $A$, Rönnemaa $T$, Lehtimäki $T$, Kähönen $M$, et al. Branched-chain and aromatic amino acids are predictors of insulin resistance in young adults. Diabetes Care. 2013;36:648-55.

9. Wang T, Larson M, Vasan R, Cheng S, Rhee E, McCabe E, et al. Metabolite profiles and the risk of developing diabetes. Nat Med. 2011;17:448-53.

10. Kume S, Araki S, Ono N, Shinhara A, Muramatsu T, Araki H, et al. Predictive properties of plasma amino acid profile for cardiovascular disease in patients with type 2 diabetes. PLoS One. 2014;9(6):e101219.

11. Mangge $H$, Zelzer S, Prüller F, Schnedl WJ, Weghuber D, Enko D, et al. Branched-chain amino acids are associated with cardiometabolic risk profiles found already in lean, overweight and obese young. J Nutr Biochem. 2016;32: $123-7$.

12. Yamakado M, Nagao K, Imaizumi A, Tani M, Toda A, Tanaka T, et al. Plasma free amino acid profiles predict four-year risk of developing diabetes, metabolic syndrome, dyslipidemia, and hypertension in Japanese population. Sci Rep. 2015; 5:11918.

13. Yamakado M, Tanaka T, Nagao K, Ishizaka Y, Mitushima T, Tani M, et al. Plasma amino acid profile is associated with visceral fat accumulation in obese Japanese subjects. Clin Obes. 2012;2:29-40.

14. Miyagi Y, Higashiyama M, Gochi A, Akaike M, Ishikawa T, Miura T, et al. Plasma free amino acid profiling of five types of cancer patients and its application for early detection. PLoS One. 2011;6(9):e24143.

15. Shimbo K, Oonuki T, Yahashi A, Hirayama K, Miyano H. Precolumn derivatization reagents for high-speed analysis of amines and amino acids in biological fluid using liquid chromatography /electrospray ionization tandem mass spectrometry. Rapid Commun Mass Spectrom. 2009;23:1483-92

16. Shimbo K, Yahashi A, Hirayama K, Nakazawa M, Miyano H. Multifunctional and highly sensitive precolumn reagents for amino acids in liquid chromatography/tandem mass spectrometry. Anal Chem. 2009;81:5172-9.

17. Newgard CB. Interplay between lipids and branched-chain amino acids in development of insulin resistance. Cell Metab. 2012;15:606-14.

18. Chiu KC, Chuang LM, Yoon C. Comparison of measured and estimated indices of insulin sensitivity and beta cell function: impact of ethnicity on insulin sensitivity and beta cell function in glucose-tolerant and normotensive subjects. J Clin Endocrinol Metab. 2001;86:1620-5.

19. Lim U, Ernst T, Buchthal SD, Latch M, Albright CL, Wilkens LR, Kolonel LN, Murphy SP, Chang L, Novotny R, Le Marchand L. Asian women have greater abdominal and visceral adiposity than Caucasian women with similar body mass index. Nutr Diabetes. 2011;1:e6.

20. Takashina C, Tsujino I, Watanabe T, Sakaue S, Ikeda D, Yamada A, et al. Associations among the plasma amino acid profile, obesity, and glucose metabolism in Japanese adults with normal glucose tolerance. Nutr Metab (Lond). 2016:13:5.

21. Pozefsky T, Felig P, Tobin JD, Soeldner JS, Cahill Jr GF. Amino acid balance across tissues of the forearm in postabsorptive man. Effects of insulin at two dose levels. J Clin Invest. 1969;48:2273-82.

22. Adams SH. Emerging perspectives on essential amino acid metabolism in obesity and the insulin-resistant state. Adv Nutr. 2011;2:445-56.

23. Chevalier S, Burgess SC, Malloy CR, Gougeon R, Marliss EB, Morais JA. The greater contribution of gluconeogenesis to glucose production in obesity is related to increased whole-body protein catabolism. Diabetes. 2006:55:675-81.

24. Würtz $P$, Tiainen $M$, Mäkinen VP, Kangas AJ, Soininen $P$, Saltevo J, et al. Circulating metabolite predictors of glycemia in middle-aged men and women. Diabetes Care. 2012:35:1749-56. 